\title{
Whither inhaled insulin?
}

\author{
Bob Young
}

This is an exciting time in the treatment of diabetes. Continuous, subcutaneous insulin infusion and continuous glucose monitoring technologies are making optimal glucose control accessible to more people with type 1 diabetes. Glucagon-like peptide 1 and amylin agonists, dipeptidyl peptidase IV inhibitors, and endocannabinoid receptor blockers are poised to make a similar impact on the treatment of type 2 diabetes.

Inhaled insulin is also just coming to marketone product is already licensed and several more will soon follow. Evidence from clinical trials attests to the short-term effectiveness of inhaled insulin, and patients have recorded high approval ratings. Compared with subcutaneous insulin analogs, inhaled insulin has a similar rate of onset and time to peak action, but a longer duration of action. With inhaled insulin, needles are replaced by a large inhaler device.

After inhalation of insulin, only 10\% of the original dose is absorbed; this inherent inefficiency undoubtedly contributes to the cost of inhaled insulin being greater than that of subcutaneous insulin. Evidence suggests that, in clinical trial conditions, inhaled insulin combined with appropriate basal insulin can achieve equivalent glycemia to preprandial subcutaneous insulin analogs in both type 1 and type 2 diabetes. Long-term effects on lung function or respiratory epithelium are unknown, as safety studies only extend to 2 years' duration. Smoking, asthma, and chronic obstructive pulmonary disease are absolute contraindications for inhaled insulin, and insulin-specific antibodies are found more frequently in patients taking inhaled insulin than those taking the subcutaneous form.

For type 2 diabetes, insulin is usually the last agent introduced in a treatment escalator as the disorder progresses. Patients will first use lifestyle advice (diet and exercise) with or
Evidence from clinical trials attests to the short-term effectiveness of inhaled insulin, and patients have recorded high approval ratings

$B$ Young is a

Consultant

Diabetologist at Hope Hospital, Salford, UK.

Competing interests

The author declared he has no competing interests.

www.nature.com/clinicalpractice doi:10.1038/ncpendmet0264 without oral antihyperglycemic drugs. Patients are often unenthusiastic about starting insulin because of apprehensions about needles and hypoglycemia. Care providers might also prefer to delay prescribing insulin because of the increased support required for the patient. The effectiveness of a once-daily basal insulin injection might ease both patient and provider concerns. On the other hand, prandial inhaled insulin alone can deliver target glucose control in some patients without the need for injections. Insulin resistance might mandate multiple inhaled doses with each meal. Although described as easy, administration with the current insulin inhalation device does require more dexterity than a bronchodilator inhaler, which might be of concern in elderly patients. Inhaled insulin still carries a risk of hypoglycemia and, as such, does not avoid the need for blood glucose monitoring, which can be as unpopular as injecting insulin.

In type 1 diabetes, where basal bolus treatment requires fine adjustment of mealtime insulin, it might be difficult to calibrate the inhaled dose flexibly and precisely, because the minimum amount is equivalent to three subcutaneous units. Added to this, there is a potentially confusing nonlinear relationship between dose and effect with inhaled insulin.

No doubt insulin delivery devices will improve and their costs will fall. Notwithstanding the large number of contraindicated patients, expense, and present uncertainties associated with the treatment, inhaled insulin is likely to become increasingly popular and find a place among the treatment options for people with type 2 and type 1 diabetes. But the lack of real-life patient experience, caution about safety among providers, and reluctance to endorse additional expenditure among payers means that it is still not exactly clear what that place will be. 